\title{
Systemic and clinical treatment of gingival hyperplasia associated with use of anticonvulsant
}

\author{
Tiago Martins Feijó Miguelis ${ }^{1}$, Karoline Von Ahn Pinto¹, Luis Eduardo Rilling Nova Cruz¹, \\ Juan Pablo Aitken Saavedra ${ }^{2-3}$, Josué Martos ${ }^{1^{*}}$
}

\begin{abstract}
1. Department of Semiology and Clinics, School of Dentistry. Federal University of Pelotas, Pelotas, Brazil 2. Department of Oral Pathology and Medicine, School of Dentistry, University of Chile, Santiago, Chile. 3. Post Graduate Program in Dentistry. Federal University of Pelotas. Pelotas, Brazil
\end{abstract}

* Corresponding Author: Josué Martos | Department of Semiology and Clinics, School of Dentistry, Gonçalves Chaves street. 457, Pelotas, RS 96015560, Brazil | Telefax: +55 5332224439 | E-mail: josue.sul@terra.com.br

Work received on 21/03/2018.

Approved for publication on 28/04/2018

\begin{abstract}
Anticonvulsants are drugs that can modify the gingival tissues response to inflammatory processes in the presence of dental plaque, inducing gingival overgrowth. Preexisting gingival inflammation induced by dental plaque seems to be a favorable condition to the development and/or expression of gingival overgrowth. This study describes a case in which the use of phenytoin and phenobarbital anticonvulsant associated with the presence of dental plaque provided a large and severe extent of gingival alteration. We concluded that it was possible to achieve a good result in the patient with an intensive mechanical control of dental plaque, oral hygiene orientations and substitution of the drug for other alternative medication.
\end{abstract}

KEY WORDS

Gingival hyperplasia; Phenytoin, Phenobarbital, Valproic acid.

Rev. Clin. Periodoncia Implantol. Rehabil. Oral Vol. 12(1); 50-52, 2019.

\section{INTRODUCTION}

Gingival enlargement or hyperplasia is an abnormal condition in which the gingiva presents changes in volume and ranges from a slight hyperplasia of the interdental papillae to a growth in which the dental crowns remain completely covered by the altered tissue. ${ }^{(1,2)}$ Gingival hyperplasia is not always linked to the alteration of the amount of tissue cells, which may be a consequence of alteration in the volume of the cellular constituents or of the extracellular matrix. ${ }^{(2)}$

Regarding gingival enlargement associated with medications, some systemically administered drugs are capable of modifying the inflammatory and immune response of periodontal tissues, especially gingival tissue. ${ }^{(3,4)}$

The first report of drug-associated to gingival hyperplasia was made by Kimball(5) in whom tissue change was observed after administration of an anticonvulsive drug based on phenytoin. Subsequently other drugs, such as calcium channel blockers (nifedipine) and immunosuppressant (cyclosporin), were also related to this side effect.(6) These drugs are structurally distinct, but they have in common the action of inhibiting the cellular uptake of calcium, a mechanism that is considered involved in the pathogenesis of gingival hyperplasia.

The role of plaque in gingival growth induced by anticonvulsant has been investigated, and the vast majority of studies show that the presence of plaque and gingival inflammation are important intervening factors for the development and/or expression of this clinical situation. ${ }^{(3,7)}$

The pathogenesis of gingival enlargement induced by systemic medication depends on multiple factors such as plasma concentration of the drug, genetic predisposition, presence of bacterial plaque and also a synergistic effect with other drugs. ${ }^{(8)}$ The differential expression of cytokine receptors in fibroblasts may be potentially involved in the pathogenesis of gingival enlargement secondary to the use of drugs such as phenytoin.

The aim of this study is to report the periodontal therapy carried out in a patient with anticonvulsant drugs based on phenytoin and phenobarbital presenting generalized and severe gingival growth.

\section{CASE REPORT}

A 24-year-old female patient was referred to the College of Dentistry at Federal University of Pelotas presenting oral bleeding and difficulty feeding. The clinical history revealed that the patient has epilepsy and as a consequence, used $100 \mathrm{mg}$ phenytoin and $100 \mathrm{mg}$ phenobarbital every 12 hours, as anticonvulsant drugs like additive pharmacodynamic interaction for the antiepileptic effect. The patient used, although in a precarious and difficult way, a toothbrush and toothpaste for daily oral hygiene. Severe oral malodor was present, which prevented the patient from socializing.
Clinical examination presents a lobulated aspect of the gingiva with severe gingival enlargement with clinical crowns covered to a large extent, showing a bucco-lingual dimension greater than $3 \mathrm{~mm}$ and a depth of probing greater than $6 \mathrm{~mm}$ (Figure 1). On palpation the gingiva was firm and resilient in consistency. Clinically, the patient still had a visible plaque and visible areas of gingival bleeding (Figure 2a). In the radiographic examination there were no horizontal bone resorptions, either localized or generalized (Figure $2 b$ ).

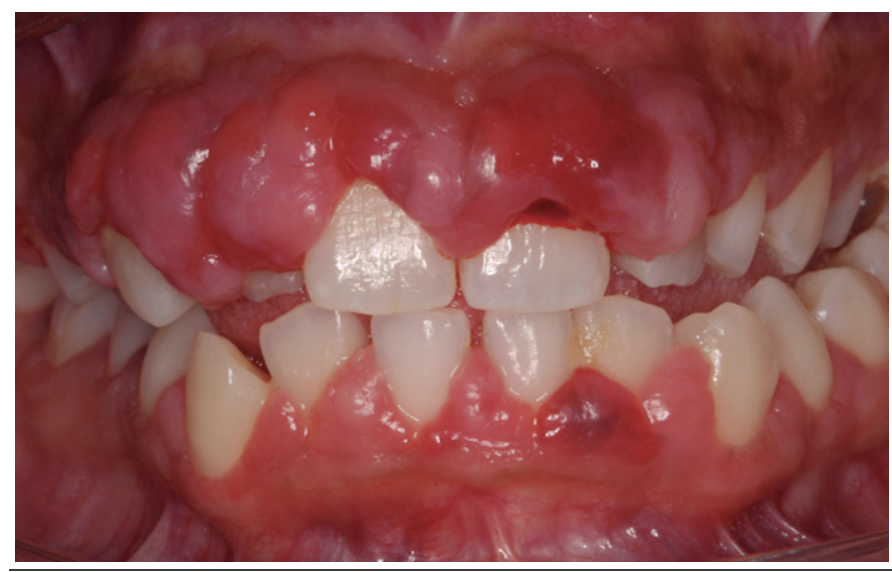

Figure 1. Clinical appearance of phenytoin-associated gingival overgrowth.

No similar history of gingival enlargement of any family member was detected during the anamnesis and the laboratory investigations showed blood counts within the normal parameters.

In possession of all clinical, radiological and medical informations, the patient was diagnosed with drug-induced gingival enlargement, aggravated by the accumulation of dental biofilm. Due to the extreme gingival bleeding, meticulous oral care was promptly instituted while awaiting evaluation by the neurologist. The patient was also advised to use $0.12 \%$ chlorhexidine digluconate mounthwash twice daily.

The neurologist was promptly consulted for details of the patient's case and systemic conditions. The treatment plan was divided into three stages; the first one associated with the systemic control of the convulsive condition with the substitution of the medication previously reported for $125 \mathrm{mg}$ valproic acid three times a day, by its neurologist and a second 


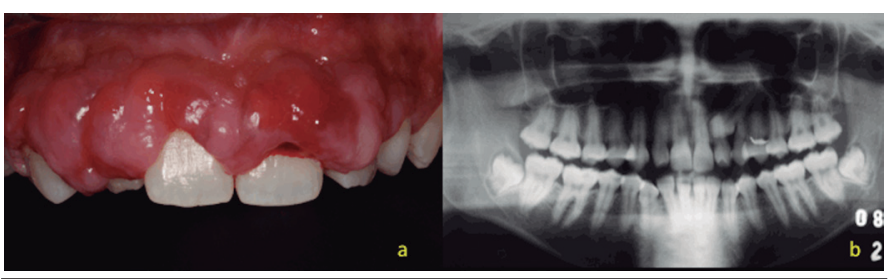

Figure 2. (a) Maxillary aspect of the initial case. (b) 1. Orthopantomogram radiograph.

stage of planning, family counseling and plaque control with supra and subgingival scraping sessions. A final planning step established that if necessary, a gingival regrowth surgery would be performed. In addition to the interdental brush, concomitant use of $0.12 \%$ chlorhexidine digluconate solution twice daily was recommended as an aid to mechanical control.

The patient presented regression of gingival growth and no bleeding after the first weeks of local and systemic treatment. The maxillary and mandibular teeth presented their crowns intact and without mobility after an interval of two months. Maintenance appointments occurred every fortnight in the first semester of treatment and every 30 days from the second semester, when deplacement procedures and oral hygiene instructions were performed (Figure 3a, 3b, 3c, 3d).

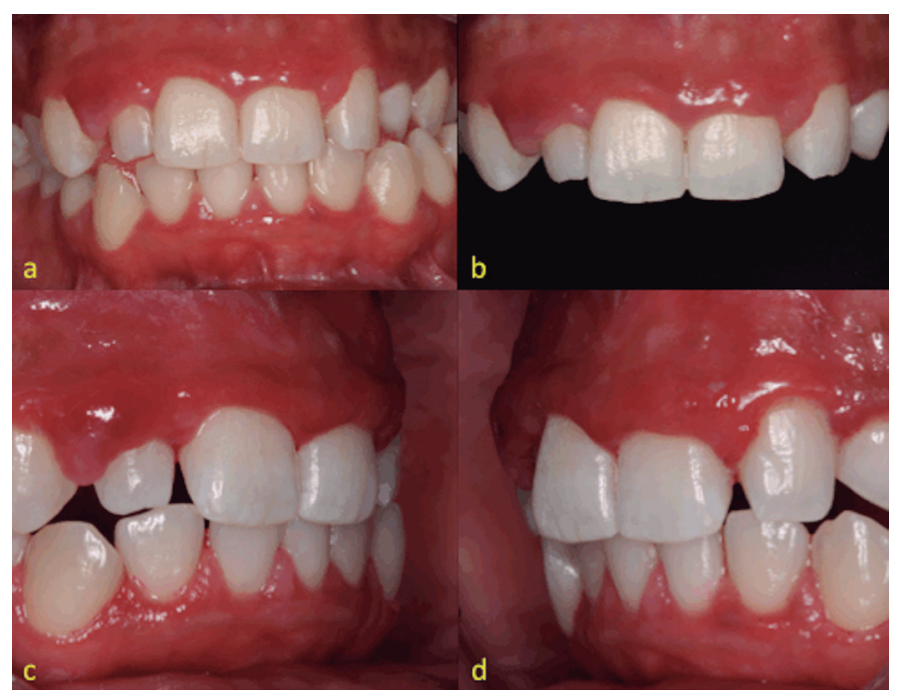

Figure 3. Gingival aspect of maxillary and mandibular 14-months after initial treatment (a) Frontal view. (b) Approximate maxillary aspect. (c) Right side view. (d) Left side view.

After 18 months of clinical follow-up, although the patient presented mild hyperplasia in the upper lateral incisor, as a result of poor dental position, she presented adequate oral hygiene proven by auxiliary methods such as dental plaque disclosing agent, in addition to periodontal probing (Figure 4a, 4b). There was no recurrence of gingival growth and absence of regions with gingival bleeding after periodontal probing (Figure 4c, 4d). The patient is undergoing medical treatment with no systemic changes due to the change in anticonvulsive drug therapy and an orthodontic treatment plan for realignment and bite descrucing in the upper right quadrant was instituted (Figure 5a, 5b, 5c, 5d).

Oral hygiene maintenance has improved a lot, which has resulted in absence of malodor, thereby significantly increasing the patient's quality of life. The actual results were found to be extremely satisfactory both esthetically and functionally.

\section{DISCUSSION}

The gingival hyperplasia, with its potential aesthetic implication complicated by inflammatory changes due to plaque accumulation, presents an affliction for patients and health professionals. The reported case is an exemplification of a progressive gingival enlargement caused by a drug-induced and complicated by inflammatory changes due to plaque accumulation.

Generally, the treatment plan in cases like the one presented, consist of conservative therapy with education on oral health, meticulous oral hygiene instruction and motivation in combination with scaling and root planing. ${ }^{(9)}$ The use of a chlorhexidine solution has been shown to be a highly beneficial adjunctive regimen to mechanical oral hygiene

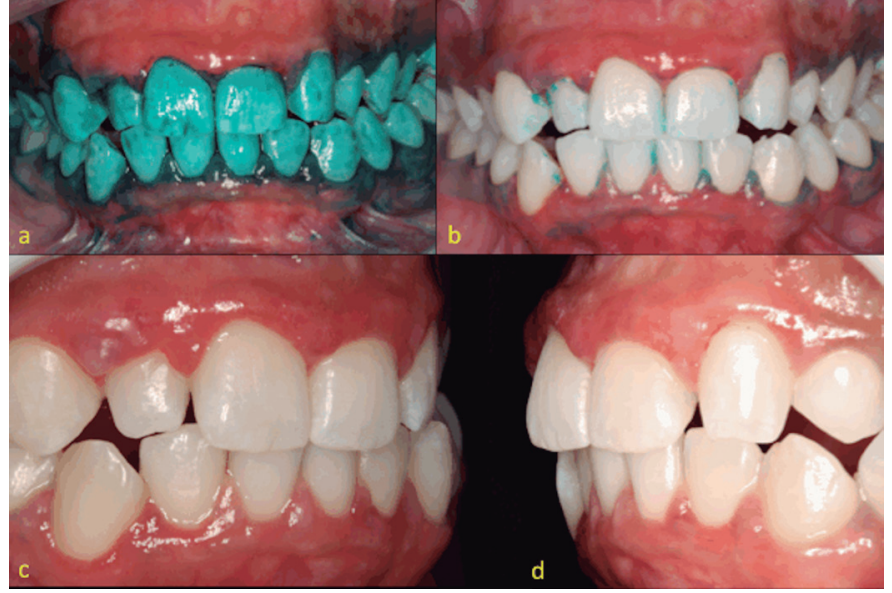

Figure 4. (a) Plaque-Identifying in frontal view. (b) Immediate aspect after vigorous washing with water. (c) Right appearance after prophylaxis. (d) Left appearance after prophylaxis.

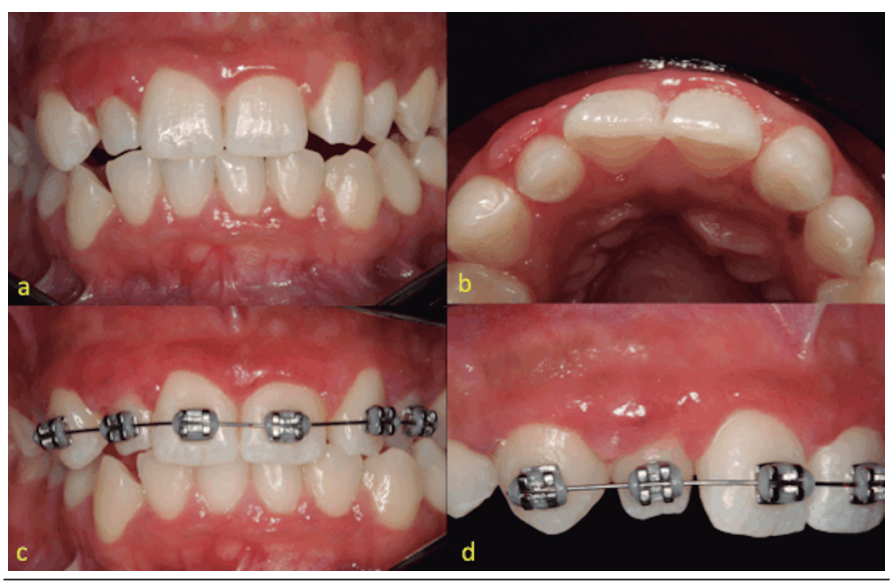

Figure 5. (a) A 24-month periodontal follow-up. (b) Mild hyperplasia in the upper lateral incisor, as a result of malposition. (c) Orthodontic treatment for realignment and bite descrucing in the upper right quadrant. (d) Labial view.

methods. ${ }^{(10)}$ Besides this, in clinical problem presented, the establishment of an integrated treatment plan involving dental professionals and the neurologist was the key point for the resolution, starting with the substitution of the medication previously used.

All stages of the established treatment plan were discussed with the patient and family so that there was no misunderstanding of information, especially at the stage where the patient's adherence would be a key and essential point for treatment. Fortunately we have succeeded in implementing the three phases of the established treatment plan.

Inflammatory changes in the gingival tissues appear to be a significant determinant of gingival hyperplasia, suggesting that inflammatory mediators influence the entire sequence of events. ${ }^{(11)}$ There is an interaction between the drug and/or its metabolite and the gingival fibroblasts that seems to increase by gingival inflammation. The authors suggest that the interaction between the drug and the gingival fibroblasts is calcium-dependent. In addition, drugs that affect intracellular calcium metabolism or transport may stimulate gingival fibroblasts. ${ }^{(12)}$

In the clinical case described, we would have a grade 4, according a new classification index for gingival overgrowth. ${ }^{(13)}$ At this classification, a severe gingival increase with extensive gingival thickening was observed, the clinical crown being covered to a large extent, having a bucco-lingual dimension greater than $3 \mathrm{~mm}$ and a depth of probing greater than $6 \mathrm{~mm}$.

Ideally, the best approach for gingival enlargment induced by the medication would be the discontinuation of the associated medication used. However, this intervention is often not possible because of the need to treat the epilepsy.(14) The phenytoin and phenobarbital medications used for the treatment of epilepsy of the patient were replaced effectively by valproic acid. Accounts of similar clinical cases, had already described phenytoin-induced gingival overgrowth. ${ }^{(15)}$

The patient responded very well with the substitution of previously used drugs. With regard to medication, some researchers suggested that sodium valproate may be considered a safe alternative, regarding 
the periodontal aspects, to phenytoin for the treatment of adult-onset epilepsy. ${ }^{(16)}$

Among the anticonvulsants, gingival enlargement is seen mostly with phenytoin (diphenylhydantoin). The other anticonvulsants that have the same effect are vigabatrin, sodium valproate, primidone, and phenobarbital. ${ }^{(17)}$ According to a report by American Academy of Periodontology, occurrence of gingival overgrowth due to sodium valproate is rare.(18) It has been described that gingival enlargement is documented in more that $40 \%$ of patients on valproate monotherapy compared to $16 \%$ of patients on phenobarbital. ${ }^{(19)}$ It should also be considered that, although the case presented evolved satisfactorily, some cases have been successfully resolved without the need to change the drug but using complementary therapies. ${ }^{(15)}$

When all the evidences are considered, there appears to be three significant factors that are important in the expression of these gingival changes, namely, drug variables, plaque-induced inflammatory changes in the gingival tissues, and genetic factors that determine the heterogeneity of the fibroblast. ${ }^{(8)}$ Care such as professional prophylaxis should be performed frequently during drug administration. Frame regression and improvement of clinical signs generally occur four weeks after drug replacement and with home oral care. However individuals who show clinical signs or symptomatology for a long time may experience partial regression of the change. In cases where the substitution of the drug is not possible, or even after the change, the professional only observes partial regression, gingival remodeling becomes essential.

Finally, it is important to highlights the importance of multidisciplinary management in these cases to achieve success. Interdisciplinary treatment in epileptic patient with a drug-induced gingival hyperplasia is becoming an ever-increasing part of modern-day dental practice. ${ }^{(20)}$

\section{CONCLUSION}

Intensive mechanical control of dental plaque, oral hygiene orientations and switchover to alternative drug for other medication are the best way to control the gingival enlargement associated with medications.

\section{CONFLICT OF INTEREST:}

None.

\section{References}

1. Marshall RI, Bartold, PM. Medication induced gingival overgrowth. Oral Dis. 1998;4:130-51.

2. Souza DF, Chiapinotto GA, Martos J. Induction of gingival hyperplasia associated with the use of calcium channel blockers. South Braz Dent J. 2009;6:447-53.

3. Seymour RA, Ellis JS, Thomason JM. Risk factors for drug-induced gingival overgrowth J Clin Periodontol. 2000:27:217-23.

4. González AC, Castañeda LN, Romano PR, Schneider AR, Toro MAF, Hofer FD. Agrandamiento gingival por ciclosporina: reporte de un caso. Rev Clin Periodoncia Implantol Rehabil Oral. 2016:9:226-30.

5. Kimball CP. The treatment of epilepsy with sodium diphenylhydantoin. J Am Med Assoc. 1939;112:1244-6.

6. Rateitschak-Plüss EM, Hefti A, Lörtscher R, Thiel G. Initial observation that cyclosporin-A induces gingival enlargement in man. J Clin Periodontol. 1983;10:237-46. 7. Angelopoulos AP. A clinicopathological review. Diphenylhydantoin gingival hyperplasia: 2. Aetiology, pathogenesis, differential diagnosis and treatment. Dent J. 1975;41:275-7.

8. Seymour RA, Thomason JM, Ellis JS. The pathogenesis of drug-induced gingival overgrowth. J Clin Periodontol. 1996;23:165-75.

9. Gurgel BC, de Morais CR, da Rocha-Neto PC, Dantas EM, Pinto LP. Phenytoininduced gingival overgrowth management with periodontal treatment. Braz Dent J. $2015 ; 26: 39-43$.

10. Franco Neto CA, Parolo CC, Rösing CK, Maltz M. Comparative analysis of the effect of two chlorhexidine mouthrinses on plaque accumulation and gingival bleeding. Braz Oral Res. 2008;22:139-44.
11. Ellis JS, Seymour RA, Thomason JM, Butler TJ, Idle JR. Periodontal variables affecting nifedipine sequestration in gingival crevicular fluid. J Periodont Res. 1995;30:272-6.

12. Seymour RA. Calcium channel blockers and gingival overgrowth. Br Dent J. 1991:170:376-9.

13. Inglés E, Rossmann JA, Caffesse RG. New clinical index for drug-induced gingival overgrowth. Quintessence Int. 1999;30:467-73.

14. Lin K, Guilhoto LMFF, Yacubian EMT. Drug-induced gingival enlargement - Part II. Antiepileptic drugs: Not only phenytoin is involved. J Epilepsy Clin Neurophysiol. 2007;13:83-8

15. Arya R, Gulati S. Phenytoin-induced gingival overgrowth. Acta Neurol Scand 2012;125:149-55

16. Seymour RA, Smith DG, Turnbull DN. The effects of phenytoin and sodium valproate on the periodontal health of adult epileptic patients. J Clin Periodontol. 1985;12:413-9.

17. Hallmon WW, Rossmann JA. The role of drugs in the pathogenesis of gingival overgrowth. Periodontol 2000. 1999;21:176-96.

18. Dongari-Bagtzoglou A. Drug-Associated Gingival Enlargement. J Periodontol. 2004;75:1424-31.

19. Gurbuz T, Tan H. Oral health status in epileptic children. Pediatr Int. 2010:52:279-83. 20. Devanna R, Asif K. Interdisciplinary management of a patient with a druginduced gingival hyperplasia. Contemp Clin Dent. 2010;1:171-6. 